\title{
A Note on a Relation between the Synthetic and Individual Probabilities in the Quantitative Earthquake Prediction
}

\author{
Yukio Fujinawa \\ National Research Institute for Earth Science and \\ Disaster Prevention, Tsukuba 305, Japan
}

In the earthquake prediction problem the estimation of the occurrence probability of major earthquakes by using multiple precursory data is desirable. A relation between the synthetic probability and individual probabilities for the earthquake prediction relying on a Markov chain model is discussed. A historical earthquake sequence in northern China of about two thousands years and the results from the observation of the radon content anomaly which covered a period of about ten years are used. The following linear relation is assumed:

$$
P_{7(\mathrm{D})}^{*}=\alpha P_{3(\mathrm{D})}^{(\mathrm{M}) *} \cdot P_{3(\mathrm{D})}^{(\mathrm{R}) *},
$$

where $\alpha$ shows a coupling constant, a factor concerning degree of dependence of two precursory phenomena, $P_{7(\mathrm{D})}^{*}, P_{3(\mathrm{D}) *}^{(\mathrm{M})}, P_{3(\mathrm{D})}^{(\mathrm{R}) *}$ are the probabilities of the earthquake occurrence normalized by the interval of the time segment in a Markov chain, when both precursors, moderate-size earthquakes, a radon content anomaly appear as precursory phenomena, respectively. Analysis shows that this relation fits well to the actual data. The value of $\alpha$ is found to be about 150-170, independent of the interval of the time segment and radon content anomaly recurrence interval.

\section{Introduction}

In the earthquake prediction a synthetic approach is tried conventionally using multiple precursory phenomena (Rikitake, 1976; Aki, 1981; Utsu, 1982, 1983; Cao and Aki, 1983; Hamada, 1987; Maeda and Yoshida, 1990). Utsu (1982, 1983) and Aki (1981) evaluated the synthetic occurrence probability of an earthquake by making use of the concept of the probability gain of various precursors. Herein they relied on the approximation of independent precursors.

Utsu $(1982,1983)$ obtained a formula of the synthetic probability in terms of earthquake occurrence probabilities for individual precursory anomalies. His calculation was conducted by using the relations of six probabilities concerning the earthquake prediction problem based on the assumption of inter-dependence between anomalies of different kinds. In the preparatory stage of earthquakes, the stress increases with

Received February 5, 1991; Accepted February 27, 1992 
time in and around the fault zone, following some physical processes such as described in the dilatancy model, which causes various precursory phenomena. Then, it may be reasonable to expect that various earthquake precursory phenomena such as foreshocks, radon content anomaly, and crustal deformation evolve in relation to each other. These occurrences are mutually dependent, though degree of dependence would be different for each of the phenomena. We could construct a more efficient model for the earthquake prediction if we could include several phenomena which are highly independent.

Utsu (1982) also proposed a method of calculation of the synthetic probability for different precursors which are partially dependent. Cao and Aki (1983) introduced empirical constants to relate the synthetic probability and the conditional probabilities for multiple dependent precursors.

Fujinawa (1991) showed that the Markov chain model is suitable for studying of the time evolution of physical states (e.g., Feller, 1968) where the dependence among the different phenomena can be reasonably taken into account. The model was applied to a historical earthquake sequence over a period of approximately two thousands years in northern China. When we try to apply the method to the case of multiple precursors, we always encounter the lack of observation of the precursory data which spans long enough to be simultaneously used with the historical earthquake sequence. Fujinawa (1991) made a simulation using the Monte Carlo method, and the radon content anomaly data over a ten-year period in China (Cao and Aki, 1983) is extended by assuming the radon anomaly occurs as a Poisson process.

In this paper the relation between the synthetic probability and the individual probabilities is qualitatively discussed applying the Markov chain model to the data of the historical earthquakes and the simulated radon content anomaly.

\section{Method and Data}

The whole time period from the beginning of the study to the present is divided into segments of equal length $\Delta t$. At each segment, a state is designated according to the presence or absence of earthquakes and the precursory phenomena.

Here an earthquake sequence record in northern China (Fig. 1, from Cao and Aki, 1983 ) is used. Earthquakes are classified into four groups according to magnitude range. Large earthquakes $\mathrm{E}_{3}$ and $\mathrm{E}_{4}$ are defined as

$$
\mathrm{E}_{3}: 7 \leqq M \leqq 7 \frac{3}{4}
$$

and

$$
\mathrm{E}_{4}: 8 \leqq M \leqq 8 \frac{1}{2}
$$

Moderate-size earthquakes $E_{1}$ and $E_{2}$ with a magnitude range of

$$
\mathrm{E}_{1}: 4 \frac{3}{4} \leqq M \leqq 5 \frac{3}{4}
$$




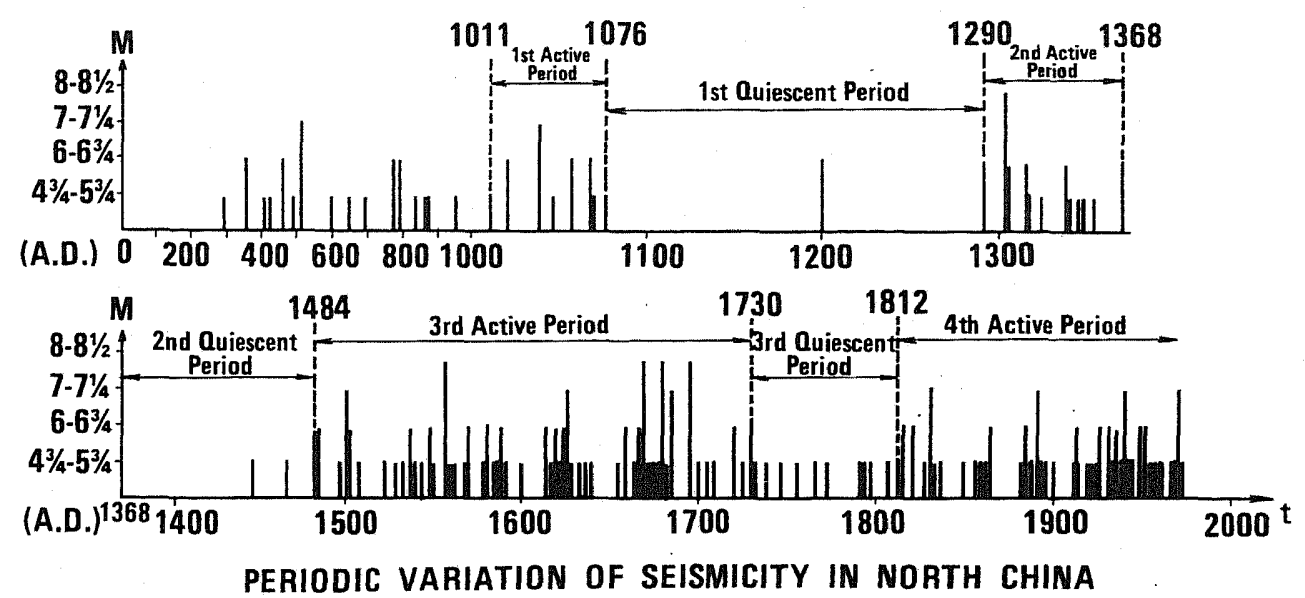

Fig. 1. The historical earthquake sequence data on northern China (from Cao and Aki, 1983) used in this study. Moderate-size earthquakes are assumed as precursors to major earthquakes.

and

$$
\mathrm{E}_{2}: 6 \leqq M \leqq 6 \frac{3}{4}
$$

are considered as precursory earthquakes as Cao and Aki (1983).

Observations of multiple precursory phenomena by the modern method have started only recently. We are going to use the radon content anomaly data. To extend the radon observation data, a simulation using the Monte Carlo method is carried out. It is reported in China that the radon content anomaly had occurred in five cases in the Haicheng area of northern China during a 10-year period (Cao and Aki, 1983). Here, without going into details about the criterion of the anomaly it will be assumed that the mean reccurrence time is two years.

It is further assumed that the radon content anomaly occurs as a Poisson process. Thus, the time interval $d$ between the successive radon content anomaly has a distribution,

$$
\begin{aligned}
& g(d)=\lambda \exp (-\lambda d), \\
& \lambda=1 / \tau,
\end{aligned}
$$

where $\tau$ denotes the recurrence interval. The value $\tau=2$ years is assigned, as stated in Cao and Aki (1983). It is also assumed that the mean recurrence time $\tau$ in the time periods prior to the earthquake are different from that in the ordinary time period, taking account of a probable change of the crustal regime due to increase of the crustal stress.

After making a time series of the radon content anomaly by the random number generation of the exponential type, each time segment was classified by seeing whether

Vol. 40, No. 3, 1992 
or not there are "earthquakes" and precursory phenomena.

In the Markov chain model with arbitrary memory length, the transition probability $P_{i, j, \cdots, n ; s}$ from the sequential states of " $i, j, \cdots, n$ " to a new state " $s$ " plays an essential role (e.g., Feller, 1968). Those probabilities can be easily evaluated when the state of each time segment is designated by the presence or absence of earthquakes and precursory phenomena.

Of most importance interest is the transition to the final state with or without the "earthquake" concerned, regardless of the other indicators at that state. Moreover, since the transition probabilities from the initial states without the earthquake are more important in the practical problem of earthquake prediction, special interest is given to the following quantities, $P_{3(D)}$ and $P_{3(S)}$, (Fujinawa, 1991), as

$P_{3(\mathrm{D})}$ : Transition probability from the state with precursory phenomena to a "disastrous" state with the "earthquake": success rate,

$P_{3(\text { (S) }}$ : Transition probability from the state with precursory phenomena to a "safe" state with the "earthquake": false alarm rate.

\section{Results}

We assume two precursors $\mathrm{A}$ and $\mathrm{B}$ are conditionally dependent but satisfy

$$
\begin{aligned}
& P(\mathrm{~A}, \mathrm{~B} \mid \mathrm{E})=a P(\mathrm{~A} \mid \mathrm{E}) P(\mathrm{~B} \mid \mathrm{E}) \\
& P\left(\mathrm{~A}, \mathrm{~B} \mid \mathrm{E}^{\mathrm{c}}\right)=b P\left(\mathrm{~A} \mid \mathrm{E}^{\mathrm{c}}\right) P\left(\mathrm{~B} \mid \mathrm{E}^{c}\right) .
\end{aligned}
$$

Here $P(A \mid E)$ is, for example, a occurrence probability of the precursory phenomena " $A$ " in the condition that the earthquake " $E$ " appears, and $E^{c}$ shows non-occurrence of the earthquake. The synthetic probability $P(\mathrm{E} \mid \mathrm{A}, \mathrm{B})$ can be approximately represented as a product of $P(\mathrm{E} \mid \mathrm{A})$ and $P(\mathrm{E} \mid \mathrm{B})$, according to the Bayes' theorem,

$$
P(\mathrm{E} \mid \mathrm{A}, \mathrm{B})=\frac{a}{b} \frac{P(\mathrm{E} \mid \mathrm{A}) P(\mathrm{E} \mid \mathrm{B})}{P(\mathrm{E}) \Delta t}
$$

for a small time interval $\Delta t$ (Cao and Aki, 1983), where $P(E)$ denotes the average frequency of occurrence of the earthquakes $E$. It is also suggested that a similar relation holds when the transition probability is used instead of the conditional probability.

An inter-relationship is to be found between the synthetic probability $P_{7(\mathrm{D})}$ and the individual probabilities $P_{3(\mathrm{D})}^{(\mathrm{R})}$ and $P_{3(\mathrm{D})}^{(\mathrm{M})}$, using the radon content anomaly simulation (Fujinawa, 1991). The probabilities were changed by changing the mean recurrence interval of the radon content anomaly $\tau_{0}$ in the ordinary period, while $\tau_{\mathrm{p}}$ in the pre-earthquake period remained fixed.

Figure 2 shows an example of simultaneous plots of $P_{7(\mathrm{D})}$ and $P_{3(\mathrm{D})}^{(\mathrm{R})}(=$ transition probability to the "dangerous" state from the state containing radon anomaly) in the case of $\tau_{\mathrm{p}}=1$ year and $\Delta t=10$ years assuming an anomalous period for the radon content for both sides of the earthquake. The crosses on the data points show the standard deviations. The deviation tends to increase with the value of the probability because the probability increase is resulted from the increase of the value $\tau_{0}$; the larger value for $\tau_{0}$ corresponds to the less frequent occurrence and causes the larger scatter 


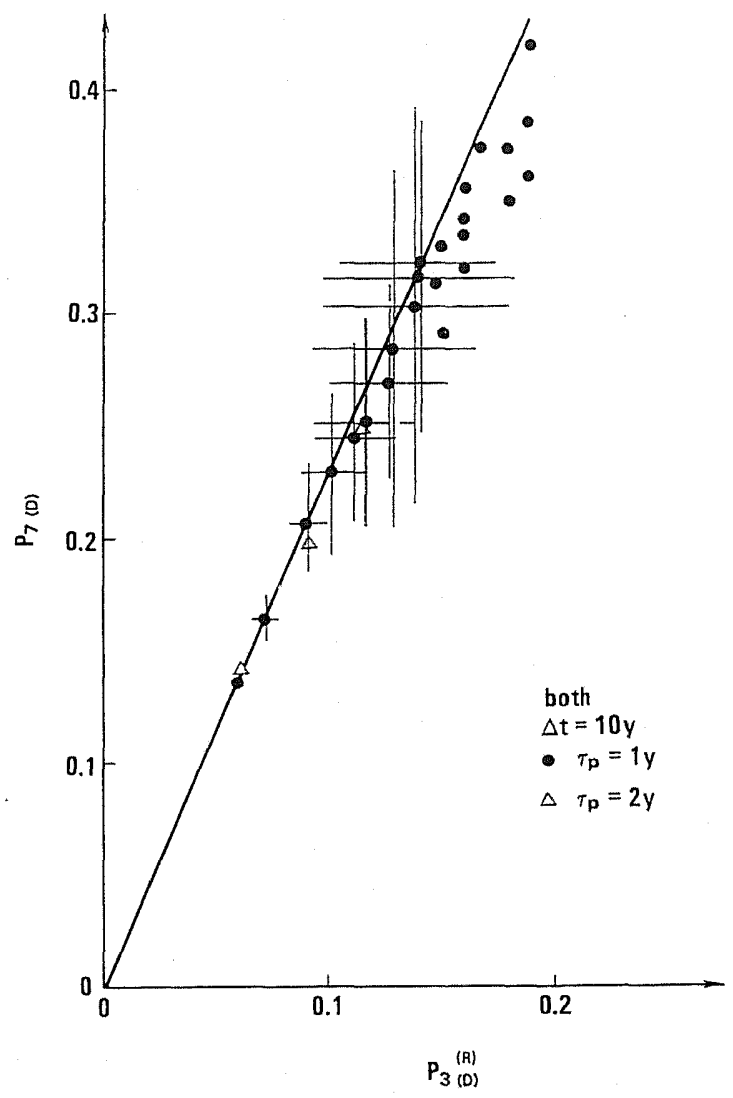

Fig. 2. Synthetic transition probability $P_{7(\mathrm{D})}$ using two precursors versus the transition probability $P_{3(\mathrm{D})}^{(\mathrm{R})}$ of the single precursor of radon content anomaly. The two cases where $\tau_{\mathrm{p}}=1$ year and $\tau_{\mathrm{p}}=2$ years are shown by fixing $\Delta t=10$ years. The period for radon anomaly was assumed before and after the large earthquake.

in the probability evaluation.

Figure 2 indicates a linear relationship between the two probabilities $P_{7(\mathrm{D})}$ and $P_{3(D)}^{(\mathrm{R})}$, and the proportional coefficient $\alpha$ turned out to be about 17 as the transition probability to the "dangerous" state from the state containing the precursory moderate-size earthquakes was $P_{3(\mathrm{D})}^{(\mathrm{M})}=0.135$. Figure 2 also shows the relation of two probabilities by assuming $\tau_{\mathrm{p}}=2$ years. The linear relationship between the two quantities can also be seen, and the value of coefficient $\alpha$ is essentially the same as in the case of $\tau_{p}=1$ year.

It is interesting to note the dependence of this relation on the time segment interval. Figure 3 shows the results for two cases of $\tau_{\mathrm{p}}=1$ year and $\tau_{\mathrm{p}}=2$ years, when $\Delta t=5$ years. The larger scatter of points in this case (though the standard deviation is not shown here) may have been induced by the small time segment interval and the limited 


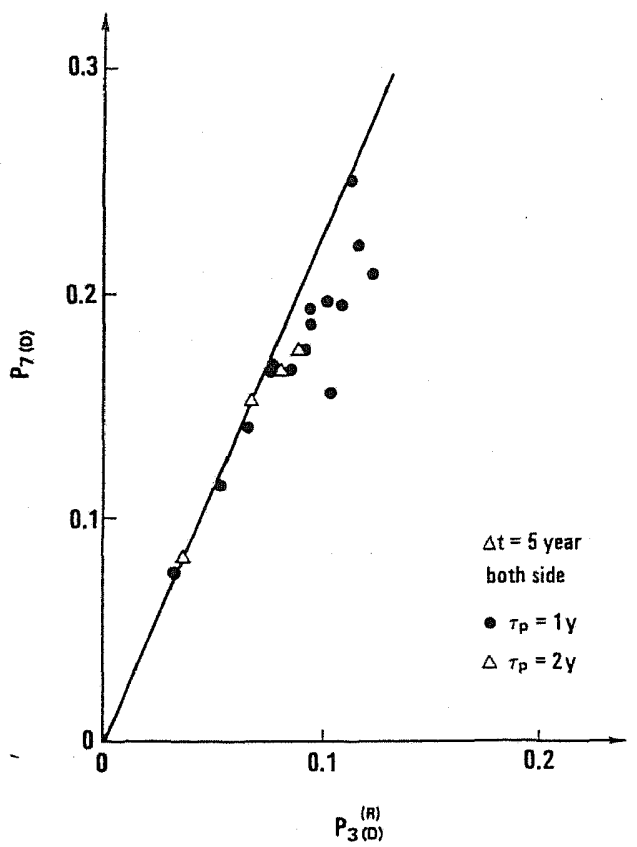

Fig. 3. Same as Fig. 2 except the time segment interval $\Delta t=5$ years.

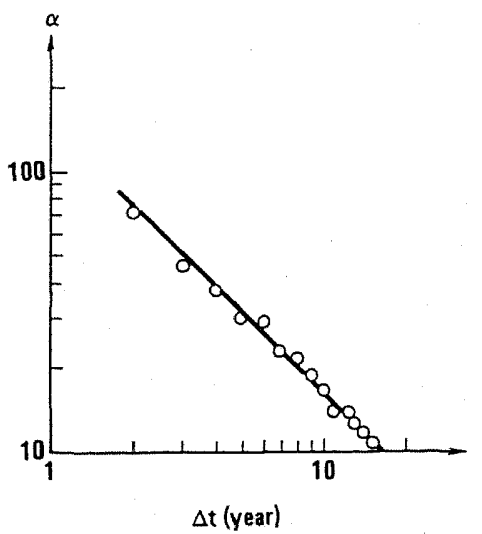

Fig. 4. Simultaneous plots of the coefficient $\alpha$ and the time segment length $\Delta t$.

number of segments. However, the overall relationship between the two quantities seems to be the same as those found in the case of $\Delta t=10$ years. Comparison of two cases also suggests that the relation does not depend on $\tau_{\mathrm{p}}$. The coefficient $\alpha$ in this case is $\alpha \fallingdotseq 30$, indicating a definite relation between the coefficient $\alpha$ and the segment interval $\Delta t$.

Figure 4 shows the relation of $\alpha$ and the time segment length $\Delta t$ in the range $\Delta t=2 \sim 30$ years, assuming $\tau_{\mathrm{p}}=1$ year for the anomalous period of the radon content 
anomaly, which is assumed to occur before and after the concerned earthquake. The data indicate the following relation,

$$
\alpha \fallingdotseq 150 / \Delta t,
$$

which agrees qualitatively with the relation of synthetic probability and two individual probabilities assumed by Cao and Aki (1983). This gives an expression using the transition probability per unit time (year) $P^{*}$ as,

$$
P_{7(\mathrm{D})}^{*} \fallingdotseq 150 P_{3(\mathrm{D})}^{(\mathrm{M}) *} \cdot P_{3(\mathrm{D})}^{(\mathrm{R}) *} \text {. }
$$

The value $\alpha$ turned out to be independent both of $\tau_{\mathrm{p}}$ and the choice of the precursor period of the radon content for both sides of the earthquake or for only the pre-earthquake period. However, the coefficient $\alpha$ would depend on the probability distribution of the radon content anomaly (here Poisson distribution was assumed) as well as that of the precursory moderate-size earthquakes (here a fixed data series of earthquakes was used). A calculation showed that,

$$
\alpha \fallingdotseq 170 / \Delta t
$$

when $\mathrm{E}_{2}$ is assumed to be the precursory earthquakes. In the case treated by Cao and Aki (1983), the proportional coefficient is $(a / b) \cdot P(\mathrm{E}) \Delta t$ as shown in (3.3). However, in the case of the transitional probability, it is not clear what the quantity $\alpha$ indicates; the value $\alpha$ may represent the rate of dependence of the two precursory phenomena.

\section{Conclusion}

In the quantitative prediction of major earthquakes, we must estimate the synthetic probability of the earthquake occurrence based on the data of multiple precursory phenomena. A relation between the synthetic probability and individual probability for each precursory phenomenon is useful to calculate the occurrence probability of earthquakes. The Markov chain model was applied to the data of a long historical record of earthquakes in northern China and the extended data of the radon content anomaly simulated by the Monte Carlo method. Transition probabilities related to the earthquake occurrence were calculated to find the relation between the synthetic probability and individual probabilities. It was found that the synthetic probability per unit time is expressed as a product of the individual probabilities with a constant coefficient, which agrees qualitatively with the proposition by Cao and Aki (1983). The coefficient is found to be independent of the mean recurrence time of the radon content anomaly and the time length of the time segment in the Markov chain model.

The author gratefully acknowledges valuable comments of two anonymous reviewers and Ms. Nishino for her typewriting.

\section{REFERENCES}

Aki, K., A probabilistic synthesis of precursory phenomena, in Earthquake Prediction: An

Vol. 40, No. 3, 1992 
International Review, Maurice Ewing Ser. 4, ed. D. W. Simpson and P. G. Richards, pp. 566-574, AGU, Washington, D.C., 1981.

Cao, T. and K. Aki, Assigning probability gain for precursors of four large Chinese earthquakes, J. Geophys. Res., 88, 2185-2190, 1983.

Feller, W., An Introduction to Probability Theory and Its Applications, Vol. 1, John Wiley \& Sons, Inc., New York, 509 pp., CH. 15, 1968.

Fujinawa, Y., A method of estimation of earthquake occurrence probability using first and multiple order Markov chain models, Natural Hazards, 4, 7-22, 1991.

Hamada, K., Statistics of precursory phenomena to earthquakes occurring in and around Japan, Proceedings of Earthquake Prediction Research Symposium, 243-249, 1987 (in Japanese).

Maeda, K. and A. Yoshida, A probabilistic estimation of earthquake occurrence on the basis of the appearance times of multiple precursory phenomena, J. Phys. Earth, 38, 431-444, 1990.

Rikitake, T., Earthquake Prediction, Elsevier, New York, 357 pp., 1976.

Utsu, T., Probabilities in earthquake prediction (The second paper), Bull. Earthq. Res. Inst., Univ. Tokyo, 57, 499-524, 1982.

Utsu, T., Probabilities associated with earthquake prediction and their relationships, Earthq. Predict. Res., 2, 105-114, 1983. 